LAWRENCE LIVERMORE N A TION AL LABORATORY

\section{Ejection of Supernova-Enriched Gas From Dwarf Disk Galaxies}

P. C. Fragile, S. D. Murray, D. N. C. Lin

June 15, 2004

The Astrophysical Journal 
This document was prepared as an account of work sponsored by an agency of the United States Government. Neither the United States Government nor the University of California nor any of their employees, makes any warranty, express or implied, or assumes any legal liability or responsibility for the accuracy, completeness, or usefulness of any information, apparatus, product, or process disclosed, or represents that its use would not infringe privately owned rights. Reference herein to any specific commercial product, process, or service by trade name, trademark, manufacturer, or otherwise, does not necessarily constitute or imply its endorsement, recommendation, or favoring by the United States Government or the University of California. The views and opinions of authors expressed herein do not necessarily state or reflect those of the United States Government or the University of California, and shall not be used for advertising or product endorsement purposes. 


\title{
Ejection of Supernova-Enriched Gas From Dwarf Disk Galaxies
}

\author{
P. Chris Fragile, Stephen D. Murray \\ University of California, Lawrence Livermore National Laboratory, P.O. Box 808, Livermore, CA 94550 \\ and \\ Douglas N. C. Lin \\ University of California, Lick Observatory, Santa Cruz, CA 95064
}

\begin{abstract}
We examine the efficiency with which supernova-enriched gas may be ejected from dwarf disk galaxies, using a methodology previously employed to study the self-enrichment efficiency of dwarf spheroidal systems. Unlike previous studies that focused on highly concentrated starbursts, in the current work we consider discrete supernova events spread throughout various fractions of the disk. We model disk systems having gas masses of $10^{8}$ and $10^{9} \mathrm{M}_{\odot}$ with supernova rates of 30, 300, and $3000 \mathrm{Myr}^{-1}$. The supernova events are confined to the midplane of the disk, but distributed over radii of 0,30 , and $80 \%$ of the disk radius, consistent with expectations for Type II supernovae. In agreement with earlier studies, we find that the enriched material from supernovae is largely lost when the supernovae are concentrated near the nucleus, as expected for a starburst event. In contrast, we find the loss of enriched material to be much less efficient (as low as $21 \%$ ) when the supernovae occur over even a relatively small fraction of the disk. The difference is due to the ability of the system to relax following supernova events that occur over more extended regions. Larger physical separations also reduce the likelihood of supernovae going off within low-density "chimneys" swept out by previous supernovae. We also find that, for the most distributed systems, significant metal loss is more likely to be accompanied by significant mass loss. A comparison with theoretical predications indicates that, when undergoing self-regulated star formation, galaxies in the mass range considered shall efficiently retain the products of Type II supernovae.
\end{abstract}

Subject headings: galaxies: dwarf — galaxies: evolution — hydrodynamics — methods: numerical - supernovae: general

\section{Introduction}

In cosmological models dominated by cold dark matter (CDM), the amplitude of fluctuations increases toward shorter wavelengths. Small galaxies are therefore the first to form, and large galaxies subsequently form through the merger of smaller systems (e.g. White \& Rees 1978; Blumenthal et al. 1984; Cole et al. 1994; Klypin, Nolthenius, \& Primack 1997; Navarro, Frenk \& White 1997). The very first generations of star formation, and hence the initial enrichment of the interstellar and intergalactic medium, therefore likely occurred within dwarf galaxies. Much recent attention has been paid to understanding the gas evolution and first generations of stars within such systems (e.g. Dekel \& Silk 1986; Katz et al. 1996; Quinn, Katz, \& Efstathiou 1986; Tegmark et al. 1987; Weinberg, Hernquist, \& Katz 1997; Mac Low \& Ferrara 1999; Sommer-Larsen, Gelato, \& Vedel 1999; Sommer-Larsen, Götz, \& Portinari 2003; Fragile et al. 2003). 
Observational work is also shedding light upon the early evolution of dwarf galaxies. Because the comoving density of damped Ly $\alpha$ systems (DLAs) at $z>2$ is comparable to that of all the ordinary matter in galactic disks today, it is tempting to identify the DLAs as the objects that evolved into the present-day $\mathrm{L}_{*}$ galaxies (Kauffmann 1996). The observationally inferred star-formation rates per unit surface area in such systems are similar to that in the Milky Way today (Wolfe, Prochaska, \& Gawiser 2003). Recent observations find that the DLA metallicity evolves significantly with redshift. The overall metallicities are, however, well below that expected from observationally inferred star-formation rates, implying a significant loss rate of enriched gas from these systems (Prochaska et al. 2003).

In addition to governing the early enrichment of the universe, star formation within dwarf systems affects their dynamical evolution as they merge to form more massive galaxies. One important issue is the "angular momentum problem." In CDM models which include only cooling, the first objects to form rapidly cool and contract. Numerical simulation show a tendency for dwarf galaxy building blocks to undergo dynamical friction and to lose a large fraction of their initial angular momentum prior to merging, leading to the formation of massive galaxies that are much more compact and have much less angular momentum than observed (Sommer-Larsen, Gelato, \& Vedel 1999). Some cosmological models have avoided this problem by invoking ad hoc star-formation rates within dwarf systems, in order to heat their gas and maintain large radii.

In earlier work, we have explored star formation that is self-consistently governed by negative feedback, in a self-regulating process. That picture has had good success in matching the star-formation rates seen in spheroids and disks (Lin \& Murray 1999, 2000), and in matching observational data for dwarf spheroidal systems (dSphs) (Dong, Lin, \& Murray 2003).

For dSphs, Dong, Lin, \& Murray (2003) find that star formation may occur in bursts. In such low mass systems, even a small burst of supernovae can lead to a substantial loss of enriched gas (Fragile et al. 2003). In more massive dwarf disk galaxies, however, where the dynamical times exceed the characteristic timescales for star formation and feedback effects, star formation is expected, in the absence of dynamical interactions between galaxies, to be a much more steady-state process.

The ejection of enriched gas from dwarf disk systems has been examined in many previous studies (De Young \& Gallagher 1990, 1994; Silich \& Tenorio-Tagle 1998; Mac Low \& Ferrara 1999; Sommer-Larsen, Götz, \& Portinari 2003). In those studies, it was found that the efficiency with which enriched material is ejected from dwarf disk galaxies may be quite high. Even in systems with gas masses as high as $10^{9} \mathrm{M}_{\odot}$ $\left(10^{10} \mathrm{M}_{\odot}\right.$ total mass), the ejection efficiency of enriched material was $97 \%$ for a supernova rate of one per 30,000 yr (Mac Low \& Ferrara 1999). This efficiency is especially striking given that, measured per unit gas mass, the supernova rates assumed are more than two orders of magnitude below that of the Milky Way today. The high ejection efficiency in the modeled dwarf systems is the result of both the shallower potential of the lower mass galaxy and the mode of star formation. In Mac Low \& Ferrara (1999), the supernova energy was input as a continuous luminosity source at the center of the model galaxies, and so the systems were unable to relax between discrete supernova events. Sommer-Larsen, Götz, \& Portinari (2003) allowed star formation to occur with high local efficiency whenever the gas density exceeded a critical value, resulting in strong bursts of star formation that also led to efficient ejection of enriched gas. These models, therefore, best represent the evolution of concentrated starbursts within the dwarf disk systems and indicate that even mild starbursts may lead to significant loss of enriched material.

In general, however, star formation is not limited to concentrated regions within young galaxies. Although it is expected to occur most strongly within regions of higher gas surface density, it shall occur 
throughout the disk, as is supported both by observational data (Gallagher \& Hunter 1987; Kennicutt 1989) and self-regulating models (Lin \& Murray 1999). Because self-regulating star formation is expected to dominate the life history of galaxies, it is of interest to see how efficiently the enriched material produced by this more quiescent process is retained.

We note here that our simulations restrict supernovae to occur in the regions of our models where the gas density is greatest, namely the midplanes. As we discuss below, this is consistent with expectations for Type II supernovae, since they occur mostly in regions of high star formation efficiency. Type I supernovae, on the other hand, which are believed to be responsible for most of the iron production within galaxies, come from older objects, which may have migrated significantly from their birthplaces and so may occur at heights well above the disk plane. In a follow up paper, we will consider the impact of Type I supernovae and the implication on the contamination of clusters of galaxies.

In this paper, we extend our earlier work on the ejection of enriched gas from dSphs (Fragile et al. 2003), by examining three-dimensional simulations of supernova energy input to dwarf disk galaxies, in which the supernovae occur as discrete events, spread out over significant fractions of the disk. We describe our numerical method and the setup of the models in $\S 2$. The results of the models are discussed in $\S 3$, and conclusions for the evolution of dwarf disk systems are discussed in $\S 4$.

\section{Numerical Method}

\subsection{Numerical Code}

The models discussed below have been computed using Cosmos, a massively parallel, multidimensional, radiation-chemo-magneto-hydrodynamics code for both Newtonian and relativistic flows developed at Lawrence Livermore National Laboratory. Tests of the Newtonian hydrodynamics options and of the internal physics relevant to the current work are presented in Anninos, Fragile \& Murray (2003) and shall not be discussed in detail here.

Because we shall be examining the effects of having multiple supernova explosions at random locations throughout the cores of dwarf galaxies, our models are run in three-dimensions on a Cartesian mesh. The simulations are run on a $256 \times 256 \times 128$ grid with a physical box size of $30 \mathrm{kpc} \times 30 \mathrm{kpc} \times 15 \mathrm{kpc}$. Each

zone, therefore, measures $117 \mathrm{pc}$ on a side. We use flat (zero-gradient) boundary conditions at each of the outer boundaries and a reflective boundary along the midplane of the disk.

The code parameters used for the present work are similar to those used previously in studying the evolution of enriched material in dwarf spheroidal galaxies (Fragile et al. 2003) and in jet-cloud interactions (Fragile et al. 2004). Chemistry is not followed in these simulations, and cooling is given by an equilibrium cooling function, assuming low metallicity $\left(Z=0.03 Z_{\odot}\right)$. In order to simulate the effects of equilibrium heating, cooling is suppressed below $10^{4} \mathrm{~K}$. Due to our inability to resolve the cooling regions behind shocks, cooling is also restricted whenever $Q / P>0.1$, where $Q$ is the scalar artificial viscosity and $P$ is the thermal gas pressure. This should help prevent overcooling in the unresolved post-shock gas, although this was not found to have a strong effect upon the evolution of dwarf spheroidal galaxy models (Fragile et al. 2003). 


\subsection{Galaxy Model}

The galaxies are modeled as non-self gravitating gas within a fixed dark-matter (DM) potential. The omission of self-gravity is reasonable, given that the baryonic-to-dark matter ratio of the systems is $\sim 0.1$. In order to facilitate comparisons with previous work, we adopt the same form for the galactic potential as used by both Mac Low \& Ferrara (1999) and Silich \& Tenorio-Tagle (2001). The spheroidal DM mass distribution is given by

$$
\rho_{d}(r)=\frac{\rho_{c}}{1-\left(r / R_{c}\right)^{2}}
$$

where the central density $\left(\rho_{c}\right)$ and scale radius $\left(R_{c}\right)$ are (Silich \& Tenorio-Tagle 2001)

$$
\begin{aligned}
\rho_{c} & =6.3 \times 10^{10}\left(\frac{M_{d}}{M_{\odot}}\right)^{-1 / 3} h^{-1 / 3} M_{\odot} \mathrm{kpc}^{-3} \\
R_{c} & =8.8 \times 10^{-6}\left(\frac{M_{d}}{M_{\odot}}\right)^{1 / 2} h^{1 / 2} \mathrm{kpc},
\end{aligned}
$$

where $M_{d}$ is the mass of the dark matter halo and $h$ is the Hubble constant in units of $100 \mathrm{~km} \mathrm{~s}^{-1} \mathrm{Mpc}^{-1}$. We adopt $h=0.65$, consistent with measurements using Type Ia supernovae (Gibson, Maloney \& Sakai 2000) and gravitational lensing (Williams \& Saha 2000), and slightly smaller than the value determined by recent Cepheid measurements (Freedman et al. 2001). The following expression gives the tidal radius of the dark matter halo $\left(R_{t}\right)$ (Mac Low \& Ferrara 1999):

$$
R_{t}=0.016\left(\frac{M_{d}}{M_{\odot}}\right)^{1 / 3} h^{-2 / 3} \mathrm{kpc} .
$$

The total mass of the halo, which is given by

$$
M_{d}=4 \pi \int_{0}^{R_{t}} \rho_{d}(r) r^{2} d r=4 \pi \rho_{c} R_{c}^{3}\left(x_{t}-\arctan x_{t}\right),
$$

where $x_{t}=R_{t} / R_{c}$, is related to the mass of the visible (gas plus stellar) matter $\left(M_{g}\right)$ by (Persic, Salucci, \& Stel 1996)

$$
\frac{M_{d}}{M_{g}} \simeq 34.7\left(\frac{M_{g}}{10^{7} M_{\odot}}\right)^{-0.29}
$$

The gravitational potential of the halo is

$$
\phi(r)=4 \pi G \rho_{c} R_{c}^{2}\left[\frac{1}{2} \ln \left(1+x^{2}\right)+\frac{\arctan x}{x}\right],
$$

where $x=r / R_{c}$. The interstellar gas density is then distributed according to (Silich \& Tenorio-Tagle 1998)

$$
\rho=\rho_{0} \exp \left[\frac{3}{2}\left(\frac{V_{e s c}}{c_{s}}\right)^{2} \chi\right]
$$

where $\rho_{0}$ is the gas density at the galactic center and $V_{e s c}=\left(2 G M_{d} / R_{t}\right)^{1 / 2}$ is the escape velocity at the halo boundary $\left(R_{t}\right)$. We adopted the equation of state of an ideal gas with cooling and heating such that the effective sound speed evolves with time. Here $c_{s}$ is the initial value of the sound speed. The function $\chi$ determines the density distribution in the (vertical) direction normal to the disk. It has the form (Silich \& Tenorio-Tagle 1998)

$$
\chi=F(r)-F(\varrho)
$$


where $\varrho$ is the cylindrical radius and

$$
F(r)=1+\frac{4 \pi \rho_{c} R_{c}^{3}}{M_{d}} x_{t}\left\{\frac{1}{2}\left[\ln \left(1+x_{t}^{2}\right)-\ln \left(1+x^{2}\right)\right]+\frac{\arctan x_{t}}{x_{t}}-\frac{\arctan x}{x}\right\} .
$$

The gas disk extends out to a cutoff radius, which depends upon the gas mass following the relation (Ferrara \& Tolstoy 2000)

$$
R_{d}=3\left(\frac{M_{g}}{10^{7} M_{\odot}}\right)^{0.338} \mathrm{kpc} .
$$

Vertically, the disk extends to a height determined by $P=P_{I G M}$, where $P=\rho c_{s}^{2}$ is the interstellar gas pressure. The value of $\rho_{0}$ in equation 8 , which sets the midplane density, is scaled to give the desired gas mass $M_{g}$ within $R_{d}$. This density distribution is chosen primarily for the purpose of comparison with other calculations. Although it does not represent that of an exponential disk, the gas profile is a reasonable approximation to dwarf galaxies. In the midplane of the galaxy, the gas is supported radially against gravity entirely by rotation. The circular velocity of the galaxy is

$$
v_{c}^{2}(r)=r \frac{\partial \phi}{\partial r}=\frac{4 \pi G \rho_{c} R_{c}^{2}}{x}(x-\arctan x) .
$$

Our models are, therefore, completely specified by choosing $M_{g}, c_{s}$, and $P_{I G M}$. In this work, all models use $c_{s}=10 \mathrm{~km} \mathrm{~s}^{-1}$ and $P_{I G M} / k=1 \mathrm{~cm}^{-3} \mathrm{~K}$. In Models 1-7, we choose $M_{g}=10^{9} M_{\odot}$, which corresponds to $M_{d} \sim 10^{10} M_{\odot}, v_{c} \sim 35 \mathrm{~km} \mathrm{~s}^{-1}, R_{c} \sim 1 \mathrm{kpc}, R_{d} \sim 14 \mathrm{kpc}$, and $R_{t} \sim 30 \mathrm{kpc}$. The gas mass in Model 8 is an order of magnitude smaller. The masses of these models are smaller than $L_{*}$ galaxies but much larger than dwarf spheroidals.

\subsection{Supernova Input}

In this work supernova events are simulated by adding either $10^{51}$ or $10^{52}$ erg of internal energy to the gas over finite regions. The internal energy is injected over an approximately spherical volume with a "top-hat" profile of 2-4 zones radius. Runs with supernova events of $10^{52}$ erg increase the chances of a single event successfully expelling enriched material from the galaxy. We justify this treatment by noting that multiple supernovae are often expected to occur in localized regions, centered upon OB associations. The effective local energy input in these regions is therefore expected to exceed that of a single supernova. However, the more energetic events can be computationally expensive to evolve for a time-explicit code such as Cosmos, especially when supernova events overlap, as occurs in either our most confined runs or highest luminosity cases. Therefore, some runs input $10^{51} \mathrm{erg}$ per event.

The supernova events are distributed randomly in space and time, subject to the following restrictions: the average rate of events is a fixed parameter within each model; all supernovae are restricted to the midplane of the galaxy; and supernovae are restricted to lie within a specified radius of the galactic center, with their distribution weighted by the enclosed area. Confining supernova events to the midplane makes it more reasonable to employ a reflective boundary as we have done. This also maximizes the probability that the galaxy will be able to contain the energy of each event and retain the enriched material. Because star formation is expected to be strongly concentrated where the gas density is greatest, limiting supernova events to the midplane is also a reasonable approximation to their actual distribution (at least for Type II supernovae).

Whenever supernova events are set off, a passive tracer is added to the same zones to which the energy is initially added. We use this tracer to track the retention of enriched material as described in $§ 4.1$. 


\section{4. $\quad$ Model Parameters}

The parameters of the models are listed in Table 1. For each model, we list the gas mass of the galaxy $\left(M_{g}\right)$, the energy input rate, the energy per supernova event, the fractional radius of the disk over which the supernovae occur $\left(R_{S N} / R_{d}\right)$, the time interval over which the supernovae are initialized, and the total simulation time.

The parameters for Model 1 are selected so as to closely match the highest mass, highest luminosity model of Mac Low \& Ferrara (1999). The primary difference is that the supernovae occur discretely in time, as is the case in all our models. In order to more closely match the results of Mac Low \& Ferrara (1999), who use a continuous input of energy, the supernova events in Model 1 have $10^{51} \mathrm{erg}$ each. The high supernova frequency helps to ensure that the nuclear gas does not have substantial time to relax between events, which should help to minimize the differences between this model and the earlier work. The duration of supernovae input phase ( $50 \mathrm{Myr}$ ) is chosen to be comparable to the average dynamical time scale (30 Myr) in the core, over which the onset of star formation is likely to spread.

Models 2-7 use the same unperturbed system as Model 1. Model 2 is exactly the same as Model 1 except that the supernovae are all set off at a single point half-way out in the disk. The scale height of the gas increases with radius, and so this model examines the ability of a non-nuclear starburst to expel enriched material, given that it must work its way through a larger volume of interstellar gas. Models 3 and 4 use the same energy input rate as Model 1 but with ten times more energy per event. In Model 3, the supernova events are spread out over $80 \%$ of the galactic disk. Thus, Model 3 represents the opposite extreme of supernova distribution. Model 4 is an intermediate case with the supernovae spread over $30 \%$ of the disk. We consider a longer duration (100 Myr) for the supernovae input phase in these two models to take into account the longer dynamical timescale and greater physical distribution of the supernovae sites. When measured per unit mass of gas within the galaxy, the supernova rates in Models 1-4 are significantly smaller than the presently observed supernova rate per unit gas mass in the Milky Way. Models 5 and 6, therefore, are similar to Models 1 and 3, except that the energy input rate is increased by an order of magnitude. In Model 7 the energy input rate is increased by yet another order of magnitude. In Model 8 the mass of the system is reduced by an order of magnitude; otherwise this model is similar to Model 3 with an energy input rate equivalent to 30 supernovae per Myr spread out over $80 \%$ of the disk. This model allows us to examine the dependence of the loss of enriched material upon galaxy mass.

In Figure 1, we plot the square root of the absolute value of the normalized gravitational potential $\left(\left|\phi-\phi_{t}\right|^{1 / 2}\right)$ as a function of radius for the two galaxy sizes considered. This function gives a good estimate of the local escape velocity. At the end of each simulation we use this to estimate the amounts of unenriched and enriched gas that will ultimately escape the galaxy, as described in $\S 4.1$. We also plot the integrated column density $\left(\Sigma=\int \rho d z\right)$ as a function of radius in the midplane of the disk. This shows that the flaring of the disk means that supernovae going of a larger radii must push through a higher column density of material than supernovae occurring near the core. This has a noticeable effect on our results.

\section{Results}

\section{1. $\quad$ Model 1}

The density and tracer evolution for Model 1 are shown in Figure 2. In this figure, the density distri-

bution of ordinary gas is illustrated by a volume visualization. Simultaneously we plot, in red, an isosurface 
of the tracer gas set at $10^{-3}$ of the input level. Similar figures are presented for each of our models.

As can be seen in Figure 2, the supernova energy input in Model 1 causes a rapid "chimneying" up the symmetry axis of the galaxy. The preferential motion of enriched gas along the rotation axis leads to a substantial loss. The time history of this loss is presented in Figure 3, alongside data from our other models. This figure tracks the cumulative amount of tracer material inside of $R_{d}$ for each model as a function of time. Each model is adjusted to the same slope and the results of the models are offset from each other for clarity. The thin solid lines show the adjusted average input rate for each model. Although this plot is useful for illustration, it does not account for enriched material that is traveling faster than the escape speed but still located within $R_{d}$. Such material is expected to eventually escape the galactic potential. We return to this point in $\S 4.1$.

In Figure 4 we plot, for each of our models, the time history of the total gas mass contained within $R_{d}$ as a function of time, normalized to the initial mass of each galaxy. Note that some mass is lost beyond $R_{d}$ in all models due simply to the fact that these galaxies are not initially in pressure equilibrium at the disk edge. That initial non-equilibrium leads to a fractional mass loss that is identical for Models 1-6, and which has nothing to do with ejection by supernovae. Only Models 7 and 8 show significantly higher mass loss than the more quiescent models. Comparing with Figure 3, we see that enriched material is preferentially lost in nearly all of these models.

Our results for Model 1 are similar, although not identical, to those of Mac Low \& Ferrara (1999). This is not unexpected, however, given that the current study uses somewhat lower resolution and a different cooling function. The fundamental conclusion, however, that highly concentrated supernovae trigger substantial loss of enriched material, remains unchanged.

\section{2. $\quad$ Model 2}

Model 2 is identical to Model 1 except that the supernovae are all initiated at a fixed point half way out in the disk. This allows us to test the dependence of our results on the location of a starburst. In Figure 5 we plot the evolution of the density and tracer for this model; it is obvious that there are significant differences from the results of Model 1. Due to the flaring of the galaxy, the starburst ejecta in Model 2 must plow through a thicker layer of gas (with higher column density) before reaching the IGM. Thus more supernova energy is expending tunneling to the surface of the galaxy, leaving less energy to eject the enriched material. The higher column density also makes cooling more efficient in the swept up material, further reducing the energy available to expel enriched material. From Figures 5 and 3, we see that, by the end of the simulation, none of the enriched material has escaped beyond $R_{d}$, in stark contrast to Model 1.

\section{3. $\quad$ Model 3}

In Model 3 the energy input rate is identical to that of Model 1, but the supernovae occur over $80 \%$ of the galactic disk. This distribution represents the opposite extremum from Models 1 and 2. Whereas those models represent the results of starburst-like activity, Model 3 represents the situation of steady, self-regulated star formation throughout the disk.

The evolution of the density and tracer for Model 3 is shown in Figure 6. As can be seen in the figure, because the supernovae are spread out over a large fraction of the disk, they only interact with a few of their 
closest neighbors. The galactic gas, therefore, has a significant amount of time to relax between supernova events. The result is that most of the enriched material is retained by the galaxy as indicated in Figure 3 . This model suggests that highly distributed star formation will be much less efficient at ejecting enriched material than more concentrated starbursts.

\section{4. $\quad$ Model 4}

Model 4 is similar to Model 3, except that the supernovae are concentrated within the inner $30 \%$ of the disk. Thus it represents an intermediate case between Models 1 and 3. The evolution of the density and tracer for this model is shown in Figure 7. From Figure 3 we see that after $100 \mathrm{Myr}$, only a small amount of tracer has been lost beyond $R_{d}$. Overall the loss of enriched material is much less than seen in Model 1. Thus, even a moderate distribution of supernova energy can significantly reduce the ejection efficiency of enriched gas.

Because the total supernova energy input in Models 3 and 4 is less than the binding energy of the gas, it might be argued that the supernovae have not had sufficient time to have a significant effect by the end of the simulations. In order to examine the possibility that continued supernova input may change our conclusions, we plot, in Figure 8, the time evolution of the internal and internal plus kinetic energies of Model 3. As can be seen, the energy of the model maintains an approximately steady-state, in which energy is radiated away at the same rate at which it is input by supernovae. We obtain very similar results for Model 4 . These models would not, therefore, evolve significantly if the simulations were carried out for longer times.

\section{5. $\quad$ Model 5}

Model 5 is similar to Model 1 except that the energy input rate is increased by an order of magnitude, to better approximates the presently observed supernova rate per unit gas mass in the Milky Way. The evolution of the density and tracer for this model is shown in Figure 9. Similar to Model 1, nearly all of the enriched material chimneys out of the galaxy along the symmetry axis, while at the same time, very little mass is lost from the remainder of the disk. Thus, our conclusion that highly concentrated starbursts result in efficient metal ejection without disturbing the bulk of the galaxy does not appear to be strongly dependent on the overall energy input rate.

\section{6. $\quad$ Model 6}

Model 6 has the same supernova rate as Model 5, yet the supernovae are spread over $80 \%$ of the disk, as in Model 3. The evolution of the density and tracer for this model is shown in Figure 10. In contrast with Model 3 (Figure 6), we see that the rate of energy input is sufficient to lead to some "tunneling," providing low densities avenues for the loss of some enriched gas, particularly at small radii. Nevertheless, as can be seen in Figures 3 and 4, the loss of tracer for Model 6 is small and there is no supernova-driven mass loss by the end of the simulation. However, we clearly see in Figure 8 that the energy input has not reached a steady state for this model. The energy input rate remains greater than the rate of loss due to radiative emission. If supernovae continue at the modeled rate, the disk will eventually be destroyed, similar to what we observe in Model 7, discussed below. 


\section{7. $\quad$ Model 7}

Model 7 is similar to Model 6 except that the energy input rate is increased by yet another order of magnitude. The evolution of the density and tracer for this model is shown in Figure 11. The supernova energy input rate of Model 7 greatly exceeds the radiative efficiency of the galaxy. The result, as can be seen in Figures 3, 4, and 11, is that after $70 \mathrm{Myr}$, nearly all of the tracer added to the galaxy so far is lost, and the disk itself is completely disrupted, leading to extensive mass loss. Since we have set the duration of the supernovae injection to be $100 \mathrm{Myr}$, the fresh replenishment maintains a residual amount of tracer elements at late epochs (see Fig. 3). This result suggests that, for supernovae distributed over a large fraction of the galaxy, a high efficiency of metal loss will be accompanied by significant overall mass loss.

\section{8. $\quad$ Model 8}

Model 8 is similar to Model 3 except that the mass of the galaxy is smaller by an order of magnitude. This model allows us to explore the dependence of our results on galaxy mass. A greater loss of enriched gas relative to Model 3 is expected for two reasons. Firstly, the potential of Model 8 is significantly shallower than that of Model 3 (Figure 1). Secondly, while the two models have same supernova energy input rate, the total radiative emission rate of Model 8 is reduced by more than an order of magnitude relative to that of Model 3 (due to the lower overall gas mass and lower gas density).

The evolution of the density and tracer for Model 8 is shown in Figure 12. As can be seen, the system is unable to relax significantly between supernova events. The gas distribution therefore becomes highly disturbed, even more so than that of Model 6, despite the fact that these two models have the same energy input rate per unit gas mass. The difference is again due both to the shallower gravitational potential of Model 8 and its reduced radiative efficiency.

The evolution of the tracer for Model 8 shows three distinct behaviors. Initially, no tracer is lost to the system. After approximately $60 \mathrm{Myr}$, tracer begins to be lost at an approximately constant rate. Late in the simulation, the tracer abundance flattens, i.e. enriched material is lost to the system at roughly the same rate it is being added. As can be seen in Figure 12, the early losses of tracer are the result of material "chimneying" near the symmetry axis. The increase in the loss rate at approximately $85 \mathrm{Myr}$, occurs when enriched material also begins to expand beyond $R_{d}$ in the equatorial plane of the disk (see the last frame of Figure 12).

\section{Discussion and Implications}

We have performed three-dimensional simulations of the evolution of enriched gas within dwarf disk galaxies. In these simulations, supernovae occur at random intervals and at random locations over prescribed fractions of the disk area. Our primary interest is to consider the efficiency of metal ejection for these systems, and its consequences for the evolution of dwarf galaxies, as we discuss below. 


\subsection{Loss of Enriched Material}

Figures 3 and 4 give a qualitative feel for the ejection history of enriched material and gas mass within our models. However, we can give a more quantitative estimate of the ejection efficiencies by considering how much material is traveling faster than the local escape speed of the dark matter halo. In this way, we define the mass-ejection efficiency as (Mac Low \& Ferrara 1999)

$$
\xi=\left(M_{g, e s c}+M_{g, l o s t}\right) / M_{g, i}
$$

where $M_{g, \text { esc }}$ is the gas mass on the grid moving at speeds greater than the escape speed, $M_{g, l o s t}$ is an

estimate of the gas mass lost from the grid at greater than the escape speed, and $M_{g, i}$ is the initial gas mass of the galaxy. Similarly, we define the metal-ejection efficiency as

$$
\xi_{Z}=\left(\mathcal{T}_{\text {esc }}+\mathcal{T}_{\text {lost }}\right) / \mathcal{T}_{S N}
$$

where $\mathcal{T}_{S N}$ is the sum of the tracer injected into each simulation and $\mathcal{T}_{\text {esc }}$ and $\mathcal{T}_{\text {lost }}$ are defined analogously to $M_{g \text {,esc }}$ and $M_{g, l o s t}$. Because our galaxies are not in initial pressure balance at the disk edge, they tend to expand sideways at the sound speed during the course of the simulations. Furthermore, since the larger galaxy models (Models 1-7) initially fill up a large fraction of the grid, they tend to lose gas off of the grid due solely to this sideways expansion. We do not include this loss in our estimate of $M_{g, l o s t}$. We evaluate $\xi$ and $\xi_{Z}$ at the end of each run $(50,75$, or $100 \mathrm{Myr})$. The results are summarized in Table 2.

The most massive galaxy model considered contains a total mass of $10^{10} \mathrm{M}_{\odot}\left(10^{9} \mathrm{M}_{\odot}\right.$ of gas $)$. For our

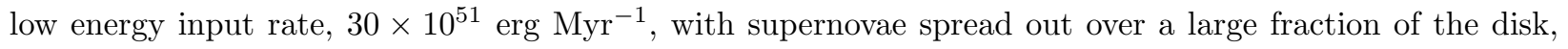
very little loss of enriched material is seen. A more significant loss is only seen if either the supernovae are concentrated over a smaller region $(\lesssim 30 \%)$ of the disk or if the supernova rate is increased by more than an order of magnitude.

In general, we find that supernovae are less effective at ejecting enriched material from dwarf disk galaxies than has been suggested in previous work. The key difference between the current and the earlier models ((Mac Low \& Ferrara 1999)) is that the earlier studies triggered supernovae either within the cores of the galaxies, or elsewhere within the systems but still with very high local efficiency. In those models, representative of starbursts, the ambient gas was unable to relax between supernova events, and enriched gas could rapidly "chimney" out of the systems. This same behavior is seen in our starburst models (1, 2 , and 5). Thus, closed-box models for the chemical evolution of these galaxies may be more appropriate when supernovae are expected to be distributed throughout the disk than when they are concentrated in the central core or bulge region.

\subsection{Comparison with Theoretical Supernova Rates}

In the self-regulated star-formation model of Lin \& Murray (1999), massive stars ionize and heat surrounding gas, preventing further star formation until the massive stars evolve off of the main sequence. This picture leads to a straightforward prediction for the formation rate of massive stars and the resulting supernova rate. From Lin \& Murray (1999), the number of massive stars within a disk out to radius $R$ is given approximately as

$$
N_{*} \sim \frac{2 \pi R^{2} H}{\frac{4}{3} \pi R_{S}^{3}}
$$


where

$$
H=2^{1 / 2} c_{s} / \Omega
$$

is the scale height of the gas, $\Omega$ is the rotational frequency at $R$,

$$
R_{S}^{3}=\frac{Q_{*}}{\frac{4}{3} \pi n^{2} \alpha_{B}}
$$

is the Strömgren sphere radius, $Q_{*}$ is the rate of emission of photoionizing photons by a massive star averaged over the population of massive stars, $n$ is the average number density of the gas, and $\alpha_{B}$ is the case B recombination coefficient (Osterbrock 1989). Using reasonable values for the above parameters, it is found that

$$
N_{*} \sim 2000 \mathrm{R}_{10}^{-7 / 2} \mathrm{M}_{10}^{5 / 2} \mathrm{~b}_{.1}^{2},
$$

where $\mathrm{R}_{10}$ is the radius of the disk in units of $10 \mathrm{kpc}, \mathrm{M}_{10}$ is the total mass of the system in units of $10^{10} \mathrm{M}_{\odot}$, and $b_{.1}$ is the gas-to-total mass ratio, in units of 0.1. An $\mathrm{L}_{*}$ galaxy, such as the Milky Way today, is predicted to have supernova rates on the order of one per century, close to the observed rate.

For galaxies such as the one used for Models 1-7, supernova rates of $\sim 100 \mathrm{Myr}^{-1}$ would be expected, intermediate to the rates applied in Models 3 and 6. It might be expected, therefore, that the loss of enriched material for a galaxy of that mass undergoing self-regulated star formation would be between $21 \%$ and $60 \%$. We note, however, that the supernova rate would not remain at this value throughout the history of the galaxy. Rather, it would decrease as the square of the gas mass, assuming that the gas scale height remained constant with time. The rate of enrichment would, therefore, decrease linearly with the gas fraction, as gas is converted into stars.

A galaxy such as that in Model 8 would be predicted to have a supernova rate $\sim 6 \mathrm{Myr}^{-1}$, several times less than applied in that model. Therefore, the loss of enriched material for such a galaxy undergoing self-regulated star formation may be significantly less than seen for Model 8 (53\%).

\subsection{Implications for Dwarf Galaxy Evolution}

In the least massive galaxies (total masses $\lesssim 10^{9} \mathrm{M}_{\odot}$ ), simulations of both disk and spheroidal systems find that enriched gas from supernovae may be largely lost to the systems (Mac Low \& Ferrara 1999; Sommer-Larsen, Götz, \& Portinari 2003; Fragile et al. 2003). Such evolution is supported by observations which find poor self-enrichment efficiency in many low-mass systems (Mateo 1998; Grebel 2001; Harbeck et al. 2001; Prochaska et al. 2003).

In more massive systems, however, our current simulations find that the supernova rates expected for self-regulated star formation do not lead to significant loss of enriched gas when the supernovae are spread over a reasonable fraction of the disk. Such galaxies would, therefore, be expected to be efficiently selfenriched. Highly efficient loss of enriched gas occurs only if star formation occurs in concentrated bursts or significantly exceed estimated self-regulated rates. In those cases, the inability of the gas to relax between supernova events allows substantial loss.

Our conclusions apply primarily to the outputs of Type II supernovae, the progenitors of which are massive, young stars. The short lifetimes of these objects do not allow for sufficient time to migrate large distances from their places of birth. Therefore, they are generally associated with regions of active star formation. In disk galaxies, these are near the midplanes, where the gas density is highest. The placement of 
the supernovae in the midplanes of our models is, therefore, a good representation to the actual distribution of Type II supernovae within galaxies. The progenitors of Type I supernovae, on the other hand, are older systems. Type I events may therefore occur further away from the disk midplane, giving them a better chance to eject enriched material from the galaxy. This is especially interesting, given that Type I supernovae are believed to be the dominant source of iron in galaxies, accounting for approximately $70 \%$ of the total production (Nomoto et al. 1984; Woosley et al. 1986; Woosley \& Weaver 1986; Matteucci 1988). It is possible that although dwarf galaxies are able to retain the heavy elements produced by Type II events, they may still be depleted in iron. In a follow up presentation, we will discuss whether this mechanism could lead to the preferential enrichment of the intergalactic medium in iron relative to the other heavy elements.

This work was performed under the auspices of the U.S. Department of Energy by University of California, Lawrence Livermore National Laboratory under Contract W-7405-Eng-48. This work is partially supported by NASA through an astrophysical theory grant NAG5-12151. 


\section{REFERENCES}

Anninos, P., Fragile, P. C., \& Murray, S. D. 2003, ApJS, 147, 177

Blumenthal, G. R., Faber, S. M., Primack, J. R., \& Rees, M. J. 1984, Nature, 311, 517

Cole, S., Aragon-Salamanca, A., Frenk, C. S., Navarro, J. F., \& Zepf, S. E. 1994, MNRAS, 271, 781

Dekel, A., \& Silk, J. 1986, ApJ, 303, 39

De Young, D. S., \& Gallagher, J. S. 1990, ApJ, 356, L15

De Young, D. S., \& Heckman, T. M. 1994, ApJ, 431, 598

Dong, S., Lin, D. N. C., \& Murray, S. D. 2003, ApJ, 596, 930

Ferrara, A., \& Tolstoy, E. 2000, MNRAS, 313, 291

Fragile, P. C., Murray, S. D., Anninos, P., \& Lin, D. N. C. 2003, ApJ, 590, 778

Fragile, P. C., Murray, S. D., Anninos, P., \& van Breugel, W. 2004, ApJ, 604, 74

Freedman, W. L. et al. 2001, ApJ, 553, 47

Gallagher, J. S. \& Hunter, D. A. 1987, AJ, 94, 43

Gibson, B. K., Maloney, P. R., \& Sakai, S. 2000, ApJ, 530, L5

Grebel, E. K. 2001, ASSS, 277, 231

Harbeck, D., Grebel, E. K., Holtzmann, J., Guhathakurta, P., Brandner, W., Geisler, D., Sarajedini, A., Dolphin, A., Hurley-Keller, D., \& Mateo, M. 2001, AJ, 122, 3092

Katz, N., Weinberg, D. H., Hernquist, L., \& Miralda-Escude, J. 1996, ApJ, 457, L57

Kennicutt, R. C. 1989, ApJ, 344, 685

Klypin, A., Nolthenius, R., \& Primack, J. 1997, ApJ, 474, 533

Lin, D. N. C., \& Murray, S. D. 1992, ApJ, 394, 523

Lin, D. N. C., \& Murray, S. D. 2000, ApJ, 540, 170

Mac Low, M.-M., \& Ferrara, A. 1999, ApJ, 513, 142

Mateo, M. 1998, ARA\&A, 36, 435

Matteucci, F. 1988, in Origin and Distribution of the Elements, ed. G. J. Mathews, (Singapore: World Scientific), 186

McWilliam, A. 1997, ARA\&A, 35, 503

Navarro, J. F., Frenk, C. S., \& White, S. D. M. 1997, ApJ, 490, 493

Nomoto, K., Thielmann, F. K., \& Yokoi, K. 1984, ApJ, 286, 644

Persic, M., Salucci, P., \& Stel, F. 1996, MNRAS, 281, 27 
Prochaska, J. X., Gawiser, E., Wolfe, A. E., Castro, S., \& Djorgowski, S. G. 2003, ApJ, 595, L9

Quinn, T., Katz, N., \& Efstathiou, G. 1996, MNRAS, 287, 49

Silich, S. \& Tenorio-Tagle, G. 1998, MNRAS, 299, 249

Silich, S. \& Tenorio-Tagle, G. 2001, ApJ, 552, 91

Sommer-Larsen, J., Gelato, S., \& Vedel, H. 1999, ApJ, 519, 501

Sommer-Larsen, J., Götz, M., \& Portinari, L. 2003, ApJ, 596, 47

Tegmark, M., Silk, J., Rees, M. J., Blanchard, A., Abel, T. \& Palla, F. 1987, ApJ, 474, 1

Weinberg, D. H., Hernquist, L., \& Katz, N. 1997, ApJ, 477, 8

White, S. D. M., \& Rees, M. J. 1978, MNRAS, 183, 341

Williams, L. L. R. \& Saha, P. 2000, AJ, 119, 439

Wolfe, A. E., Prochaska, J. X., \& Gawiser, E. 2003, ApJ, 593, 215

Woosley, S. E., Taam, R. E., \& Weaver, T. A. 1986, ApJ, 301, 601

Woosley, S. E. \& Weaver, T. A. 1986, in Radiation Hydrodynamics in Stars and Compact Objects, eds. D. Mihalas, \& K.-H. A. Winkler, (Berlin: Springer-Verlag), 91

This preprint was prepared with the AAS $\mathrm{LAT}_{\mathrm{E}} \mathrm{X}$ macros v5.0. 
Table 1. Model Galaxy Parameters

\begin{tabular}{ccccccc}
\hline \hline Model & $\begin{array}{c}M_{g} \\
\left(\mathrm{M}_{\odot}\right)\end{array}$ & $\begin{array}{c}\text { Energy input rate } \\
\left(10^{51} \mathrm{erg} \mathrm{Myr}^{-1}\right)\end{array}$ & $\begin{array}{c}\text { Energy/event } \\
\left(10^{51} \mathrm{erg}\right)\end{array}$ & $\begin{array}{c}R_{S N} / R_{d} \\
\text { SN input phase } \\
(\mathrm{Myr})\end{array}$ & $\begin{array}{c}\text { Simulation stop } \\
(\mathrm{Myr})\end{array}$ \\
\hline 1 & $10^{9}$ & 30 & 1 & 0.0 & 50 & 100 \\
2 & $10^{9}$ & 30 & 1 & $0.0^{\mathrm{a}}$ & 50 & 100 \\
3 & $10^{9}$ & 30 & 10 & 0.8 & 100 & 100 \\
4 & $10^{9}$ & 30 & 10 & 0.3 & 100 & 100 \\
5 & $10^{9}$ & 300 & 10 & 0.0 & 50 & 50 \\
6 & $10^{9}$ & 300 & 10 & 0.8 & 75 & 75 \\
7 & $10^{9}$ & 3000 & 1 & 0.8 & 100 & 100 \\
8 & $10^{8}$ & 30 & 10 & 0.8 & 100 & 100 \\
\hline
\end{tabular}

${ }^{\mathrm{a}}$ Offset from center by $R_{d} / 2$. 
Table 2. Gas and Metal Ejection Efficiencies

\begin{tabular}{ccc}
\hline \hline Model & $\xi$ & $\xi_{Z}$ \\
\hline 1 & 0.023 & 0.91 \\
2 & 0.032 & 0.47 \\
3 & 0.027 & 0.21 \\
4 & 0.028 & 0.60 \\
5 & 0.016 & 0.99 \\
6 & 0.096 & 0.60 \\
7 & 0.98 & 0.99 \\
8 & 0.064 & 0.53 \\
\hline
\end{tabular}



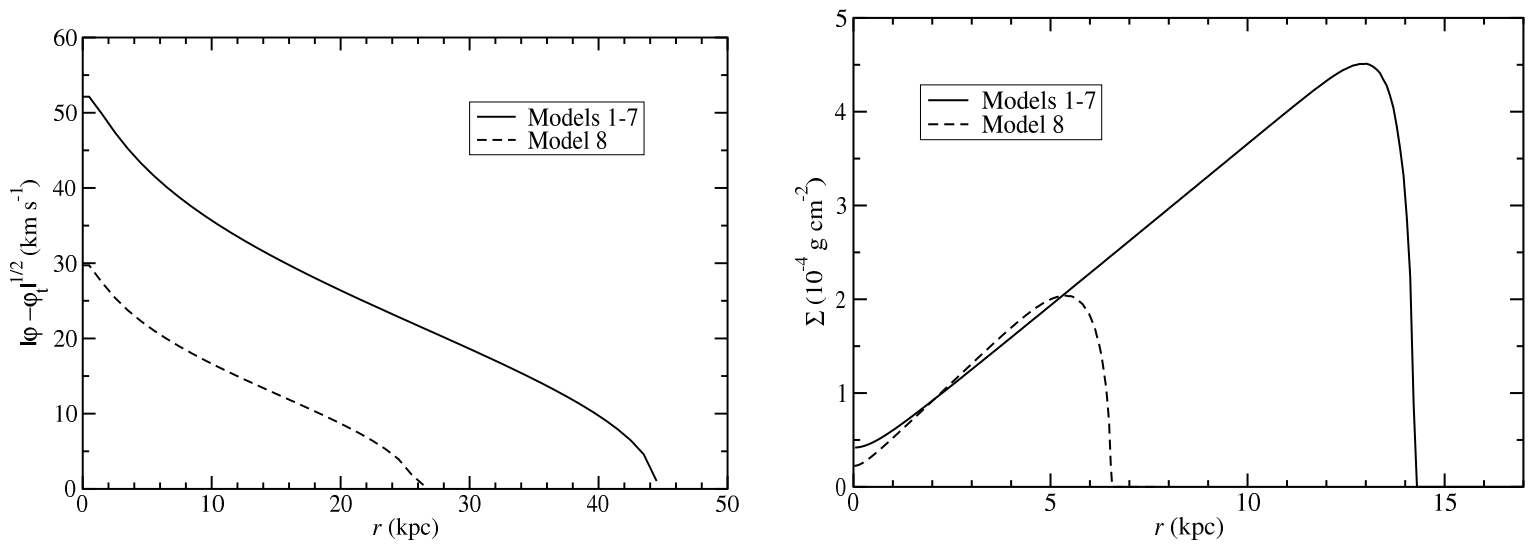

Fig. 1.- (a) Square root of the absolute value of the normalized gravitational potential as a function of radius; (b) integrated column density as a function of radius in the midplane of the disk for the galaxies in Models 1-7 (solid curves) and Model 8 (dashed curves). 


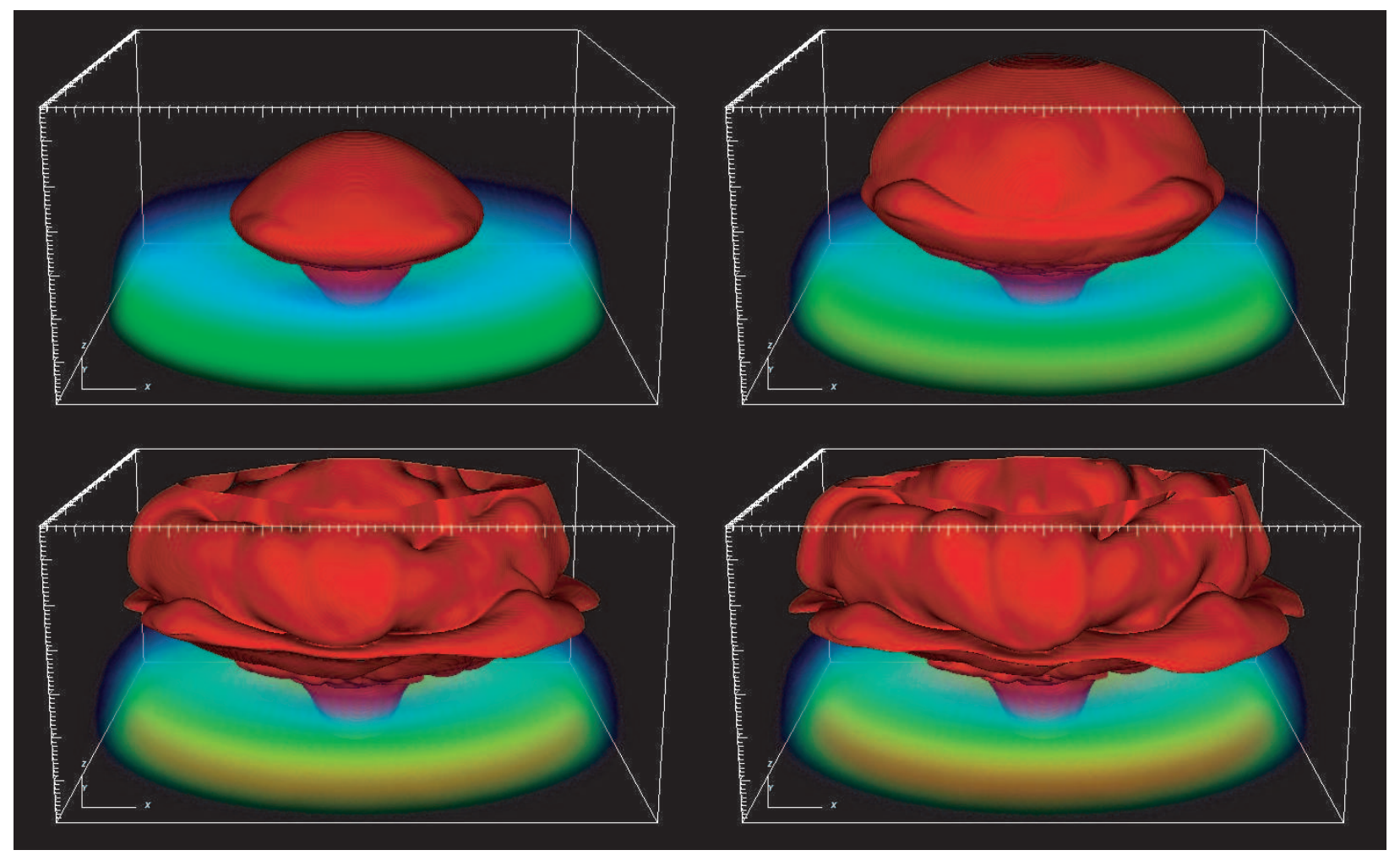

Fig. 2.- Volume rendering of gas density plus an isosurface (in red) of metal tracer for Model 1 at $t=25$, 50,75 , and $100 \mathrm{Myr}$. The metal tracer surface is set at $0.1 \%$ of the input level. 


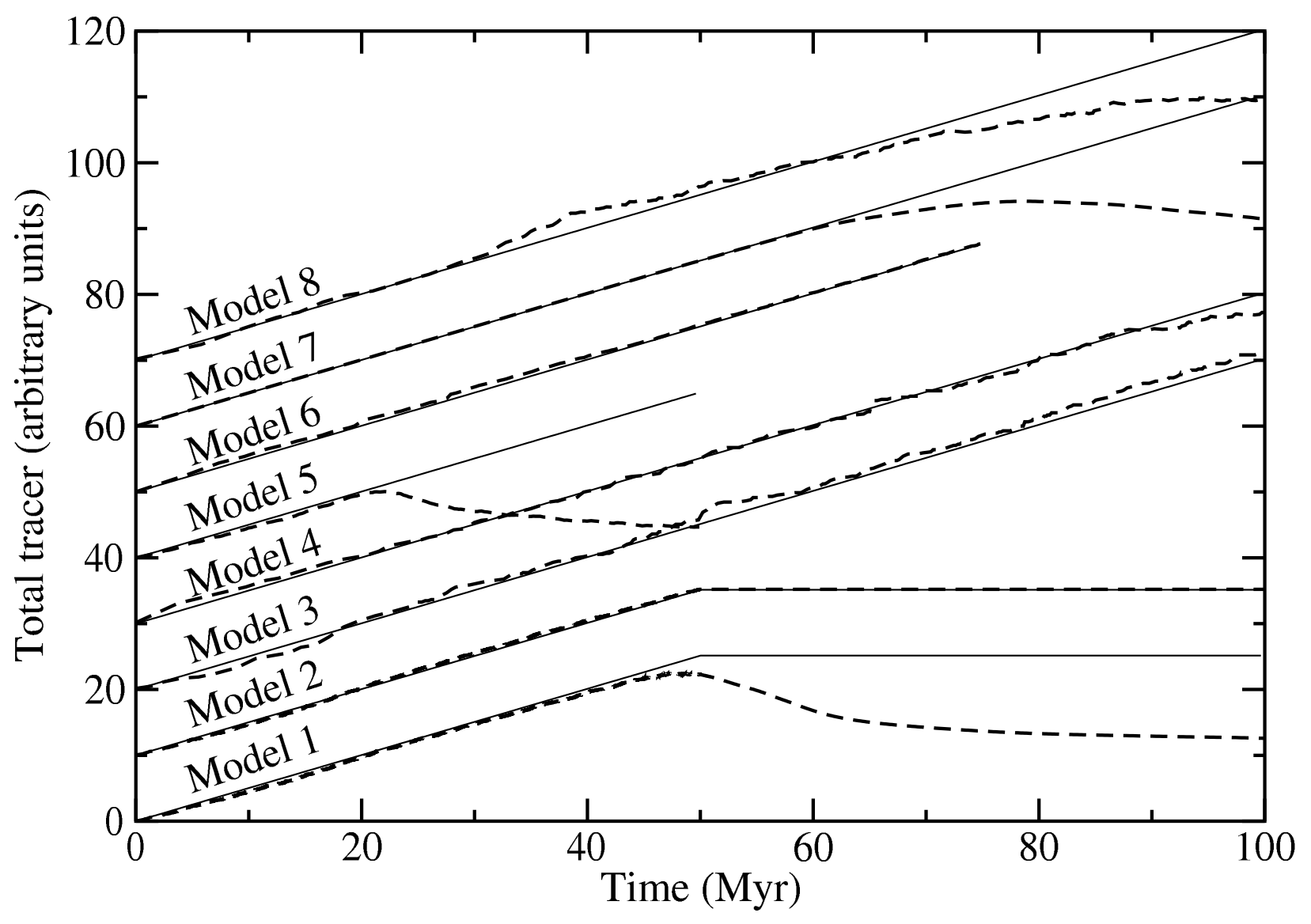

Fig. 3.- Plot of total tracer contained within $R_{d}$ as a function of time for all models. The data are adjusted to have the same slope and then offset to make the plot more legible. The thin solid lines are provided as a guide of the average tracer input rate for each model. Note that, for Models 1 and 2, supernova input ends after 50 Myr. The remaining models have continuous supernova input for the duration of the simulations. 


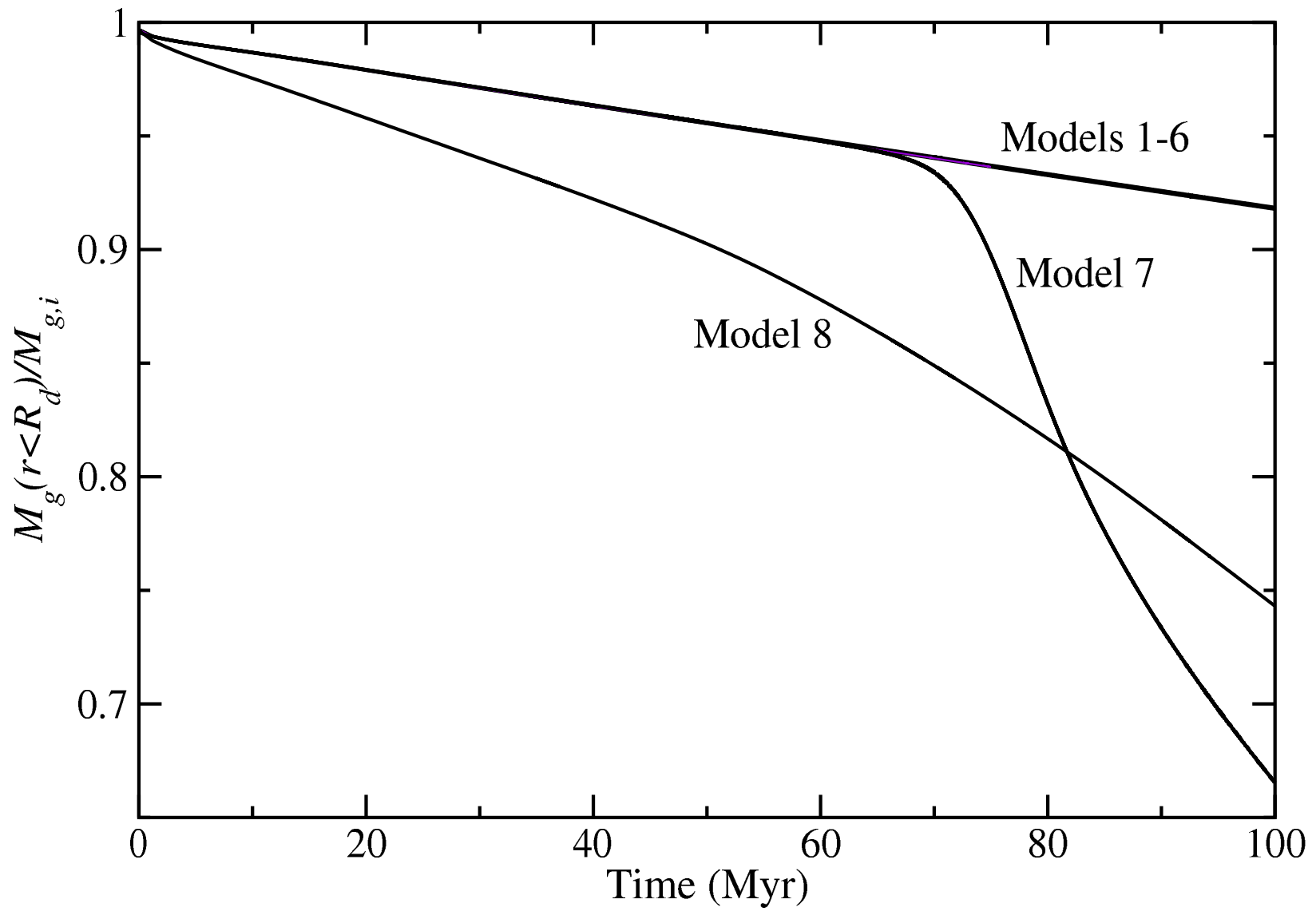

Fig. 4.- Plot of total gas mass $\left(M_{g}\right)$ contained within $R_{d}$ as a function of time for all models. The data are normalized to the initial gas mass of each galaxy. Note that some mass escapes beyond $R_{d}$ simply because the galaxies are not in initial pressure equilibrium with the background at the cut-off radius. This accounts for virtually all of the loss for Models 1-6 and the early loss in Models 7 and 8. Only Models 7 and 8 show a deviation due to the energy input of supernovae at late time $(t \gtrsim 50 \mathrm{Myr})$. 


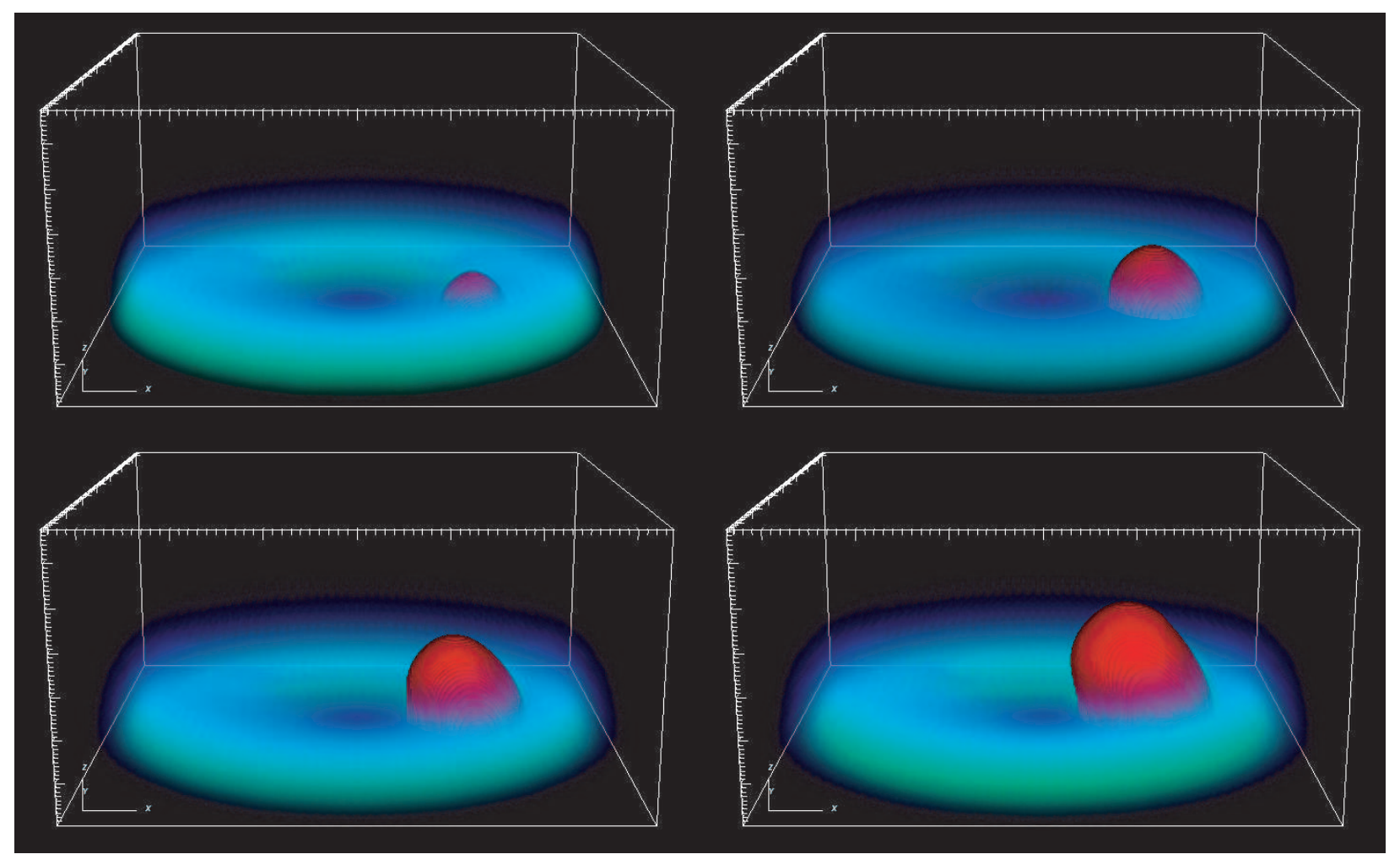

Fig. 5.- Same as Figure 2 for Model 2 at $t=25,50,75$, and 100 Myr. 


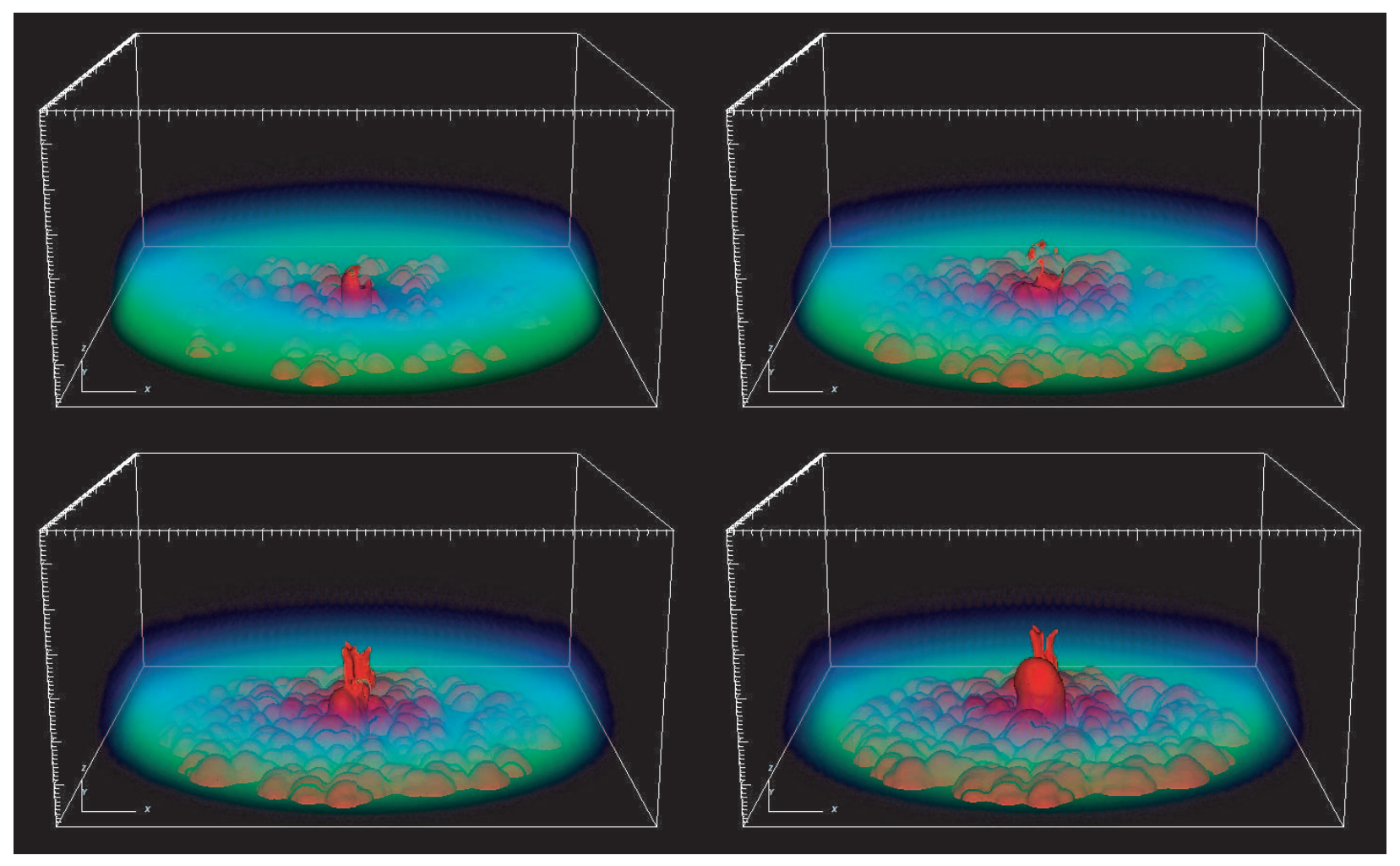

Fig. 6.- Same as Figure 2 for Model 3 at $t=25,50,75$, and 100 Myr. 


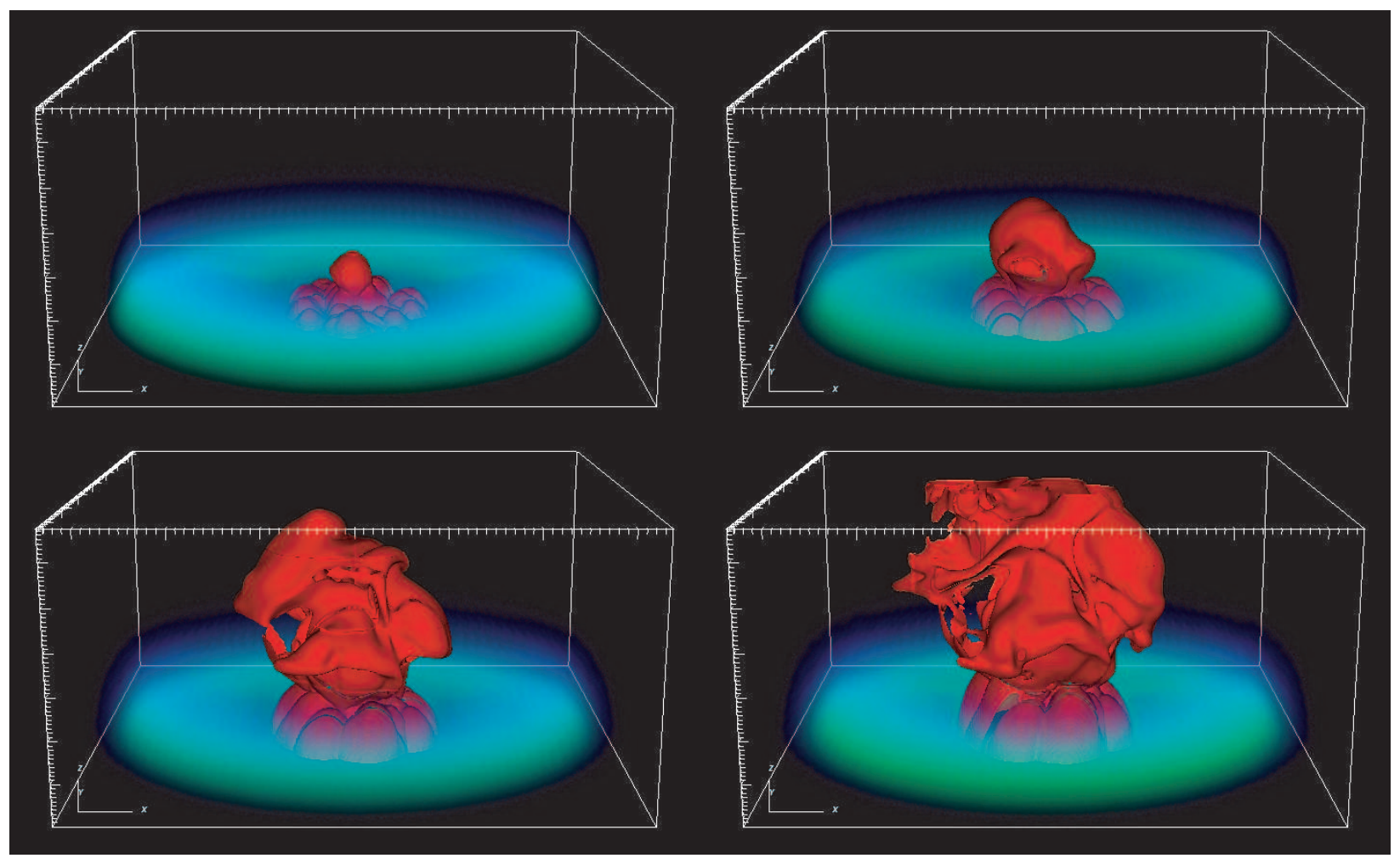

Fig. 7.- Same as Figure 2 for Model 4 at $t=25,50,75$, and 100 Myr. 


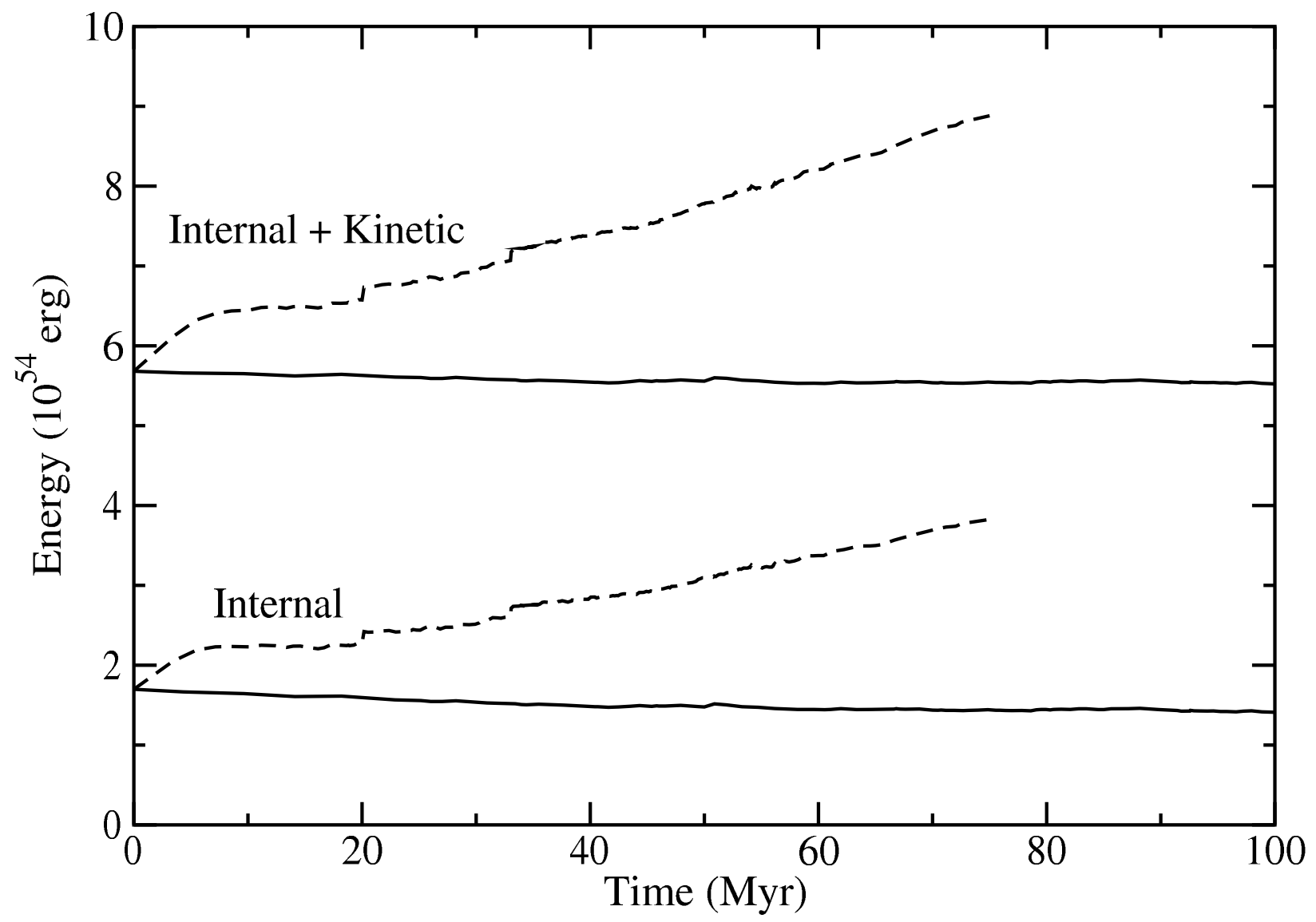

Fig. 8. - Internal and internal+kinetic energy as a function of time for Models 3 (solid line) and 6 (dashed line). 


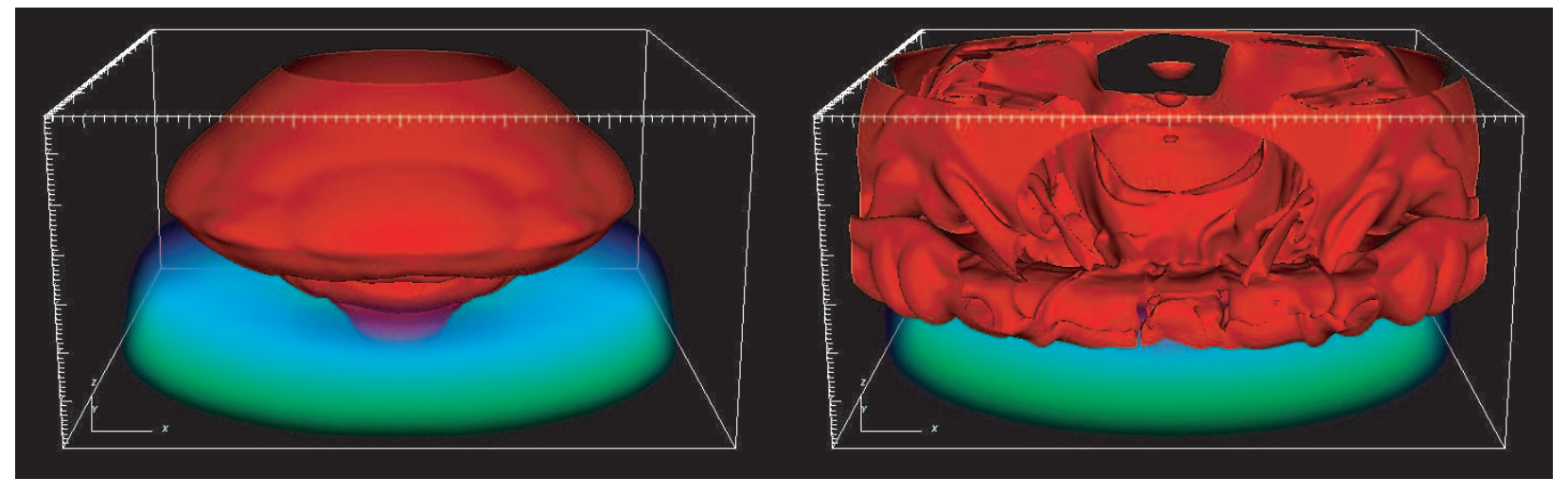

Fig. 9.- Same as Figure 2 for Model 5 at $t=25$ and 50 Myr. 


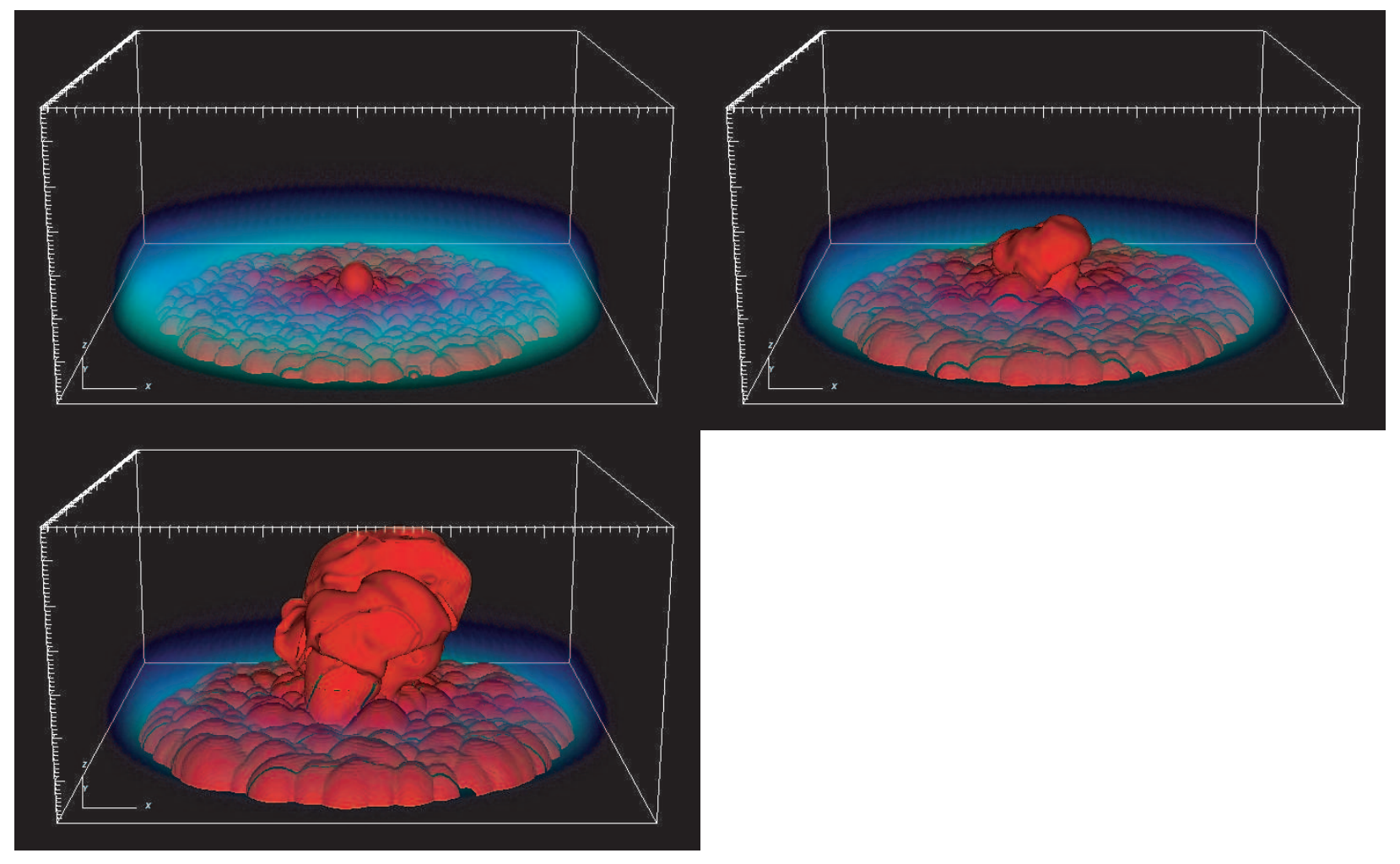

Fig. 10.- Same as Figure 2 for Model 6 at $t=25,50$, and 75 Myr. 


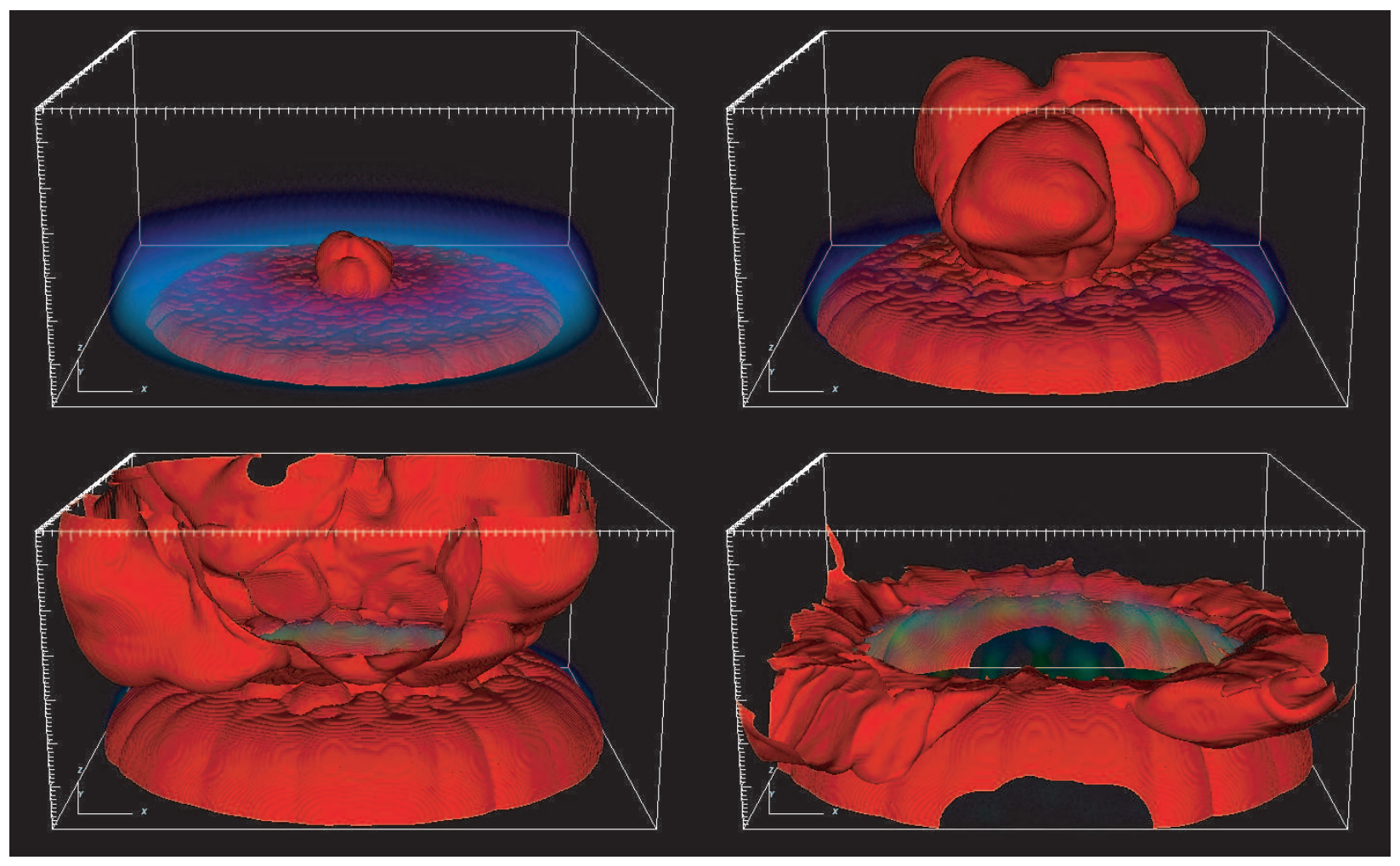

Fig. 11. - Same as Figure 2 for Model 7 at $t=25,50,75$, and $100 \mathrm{Myr}$. 


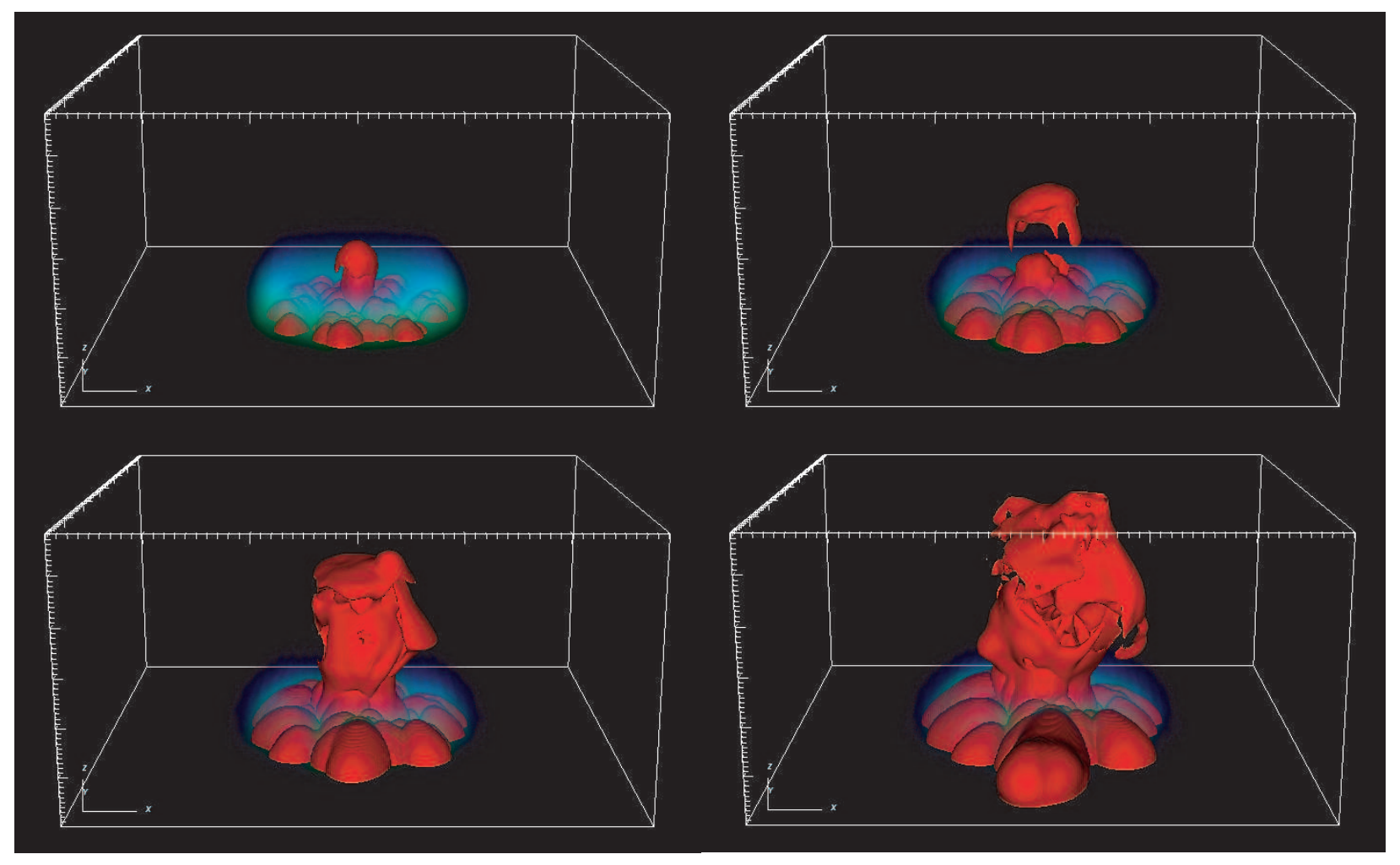

Fig. 12.- Same as Figure 2 for Model 8 at $t=25,50,75$, and $100 \mathrm{Myr}$. 